\title{
Physicochemical, morphological and cellular uptake properties of lutein nanodispersions prepared by using surfactants with different stabilizing mechanisms
}

\begin{abstract}
In this study, we prepared a series of lutein nanodispersions via the solvent displacement method, by using surfactants with different stabilizing mechanisms. The surfactants used include Tween 80 (steric stabilization), sodium dodecyl sulfate (SDS; electrostatic stabilization), sodium caseinate (electrosteric stabilization) and SDS-Tween 80 (electrostatic-steric stabilization). We then characterized the resulting lutein nanodispersions in terms of their particle size, particle size distribution, zeta potential, lutein content, flow behavior, apparent viscosity, transmittance, color, morphological properties and their effects on cell viability and cellular uptake. The type of surfactant used significantly $(p<0.05)$ affected the physical properties of the nanodispersions, but the chemical properties (lutein content) remained unaffected. Transmission electron microscopy (TEM) images obtained from this study demonstrated that the solvent displacement method was capable of producing lutein nanodispersions containing spherical particles with sizes ranging from 66.20-125.25 $\mathrm{nm}$, depending on the type of surfactant used. SDS and SDS-Tween 80 surfactants negatively affected the viability of the HT-29 cells used in this study. Thus, for the cellular uptake determination, only Tween 80 and sodium caseinate surfactants were used. The cellular uptake of the lutein nanodispersion stabilized by sodium caseinate was higher than that which was stabilized by Tween 80. All things considered, the type of surfactant with different stabilizing mechanisms did produce lutein nanodispersions with different characteristics. These findings would aid in future selection of surfactants in order to produce nanodispersions with desirable properties.
\end{abstract}

Keyword: Physicochemical; Morphological; Lutein nanodispersions; Surfactants; Stabilizing mechanisms 